Article

\title{
Decentralized and Real-Time Power Dispatch Control for an Islanded Microgrid Supported by Distributed Power Sources
}

\section{Changsun Ahn ${ }^{1, *}$ and Huei Peng ${ }^{2}$}

1 School of Mechanical Engineering, Pusan National University, 2, Busandaehak-ro 63beon-gil, Geumjeong-gu, Busan 609-735, Korea

2

Department of Mechanical Engineering, University of Michigan, 1231 Beal Ave. Ann Arbor, MI 48109, USA; E-Mail: hpeng@umich.edu

* Author to whom correspondence should be addressed; E-Mail: sunahn@pusan.ac.kr; Tel.: +81-51-510-2979; Fax: +81-51-510-0685.

Received: 3 November 2013; in revised form: 8 December 2013 / Accepted: 9 December 2013 / Published: 13 December 2013

\begin{abstract}
Microgrids can deploy traditional and/or renewable power sources to support remote sites. Utilizing renewable power sources requires more complicated control strategies to achieve acceptable power quality and maintain grid stability. In this research, we assume that the grid stability problem is already solved. As a next step, we focus on how the power can be dispatched from multiple power sources for improved grid efficiency. Isolated microgrids frequently require reconfigurations because of the grid expansion or component failures. Therefore, the control strategies ideally should be implemented in a plug-and-play fashion. Moreover, these strategies ideally require no pre-knowledge of the grid structure, and as little communication with neighboring power sources as possible. The control objective is to minimize a cost function that can be adjusted to reflect the desire to minimize energy cost and/or losses. An algorithm is designed to satisfy a derived necessary condition of function optimality. Such conditions are obtained by formulating Lagrange functions. An equivalent grid model approximates the grid structure which was later confirmed to represent the grid behavior adequately. For decentralized operations, we execute the distributed control sequentially using a simple token communication protocol. The performance of the combined system identification-Lagrange function minimization algorithm is demonstrated through simulations.
\end{abstract}


Keywords: distributed power sources; microgrid; decentralized control; optimal control; power dispatch

\section{Introduction}

Distributed renewable power sources are being deployed at a rapid pace. They promise lower environmental impacts, a reduced carbon footprint, and enhanced energy diversity and security [1-6]. These new power sources are frequently installed in rural areas far away from the main electrical grid, and sometimes forming microgrids [7-10]. The distributed power sources typically include solar cells, wind turbines, fuel cells, and are commonly augmented by traditional power sources such as diesel engines with generators. Depending on the setup, energy storage systems in the form of batteries or plug-in vehicles may be included [11-16].

In microgrid applications, the grid architecture may change more often and thus the overall integration concept needs to be more flexible to accommodate scalability and expandability [17]. The network should also be robust against issues unique to microgrids such as frequent and relatively strong perturbations in power supply and demand. The microgrid control systems thus ideally are decentralized, use simple yet robust control algorithms, and do not require much communication. In addition, the controller should not rely on a priori knowledge of the grid structure. Because of these requirements, each component should have adequate local intelligence and needs a local processor [18].

Electricity grid control is guided by two main objectives. The primary one is demand-supply balance, which has a short time horizon - the mismatch needs to be contained within predefined threshold at every instant. The secondary objective is economic dispatch, which has a longer time horizon. Given a certain total grid load, the supplies can be redistributed to reduce overall generation cost or losses by redistributing the power generations. This redistribution process does not need to happen very quickly.

To achieve a demand-supply balance in microgrids, power electronic devices control the operations of generators. These devices incorporate droop characteristics that respond to locally monitored frequency and voltage. In the literature, control strategies to maintain demand-supply balance are frequently based on conventional droop characteristics [19-23]. This study also utilizes conventional droops for the demand-supply balance, which can be implemented in a plug-and-play fashion. Moreover, they are compatible with the control strategies of high voltage lines and rotational generators. With advances in wireless communications, it becomes possible for information to be transmitted and shared. This research, therefore, studies control algorithms that use droop information solely, as well as algorithms that use a small amount of communications.

The main focus of our research — economic dispatch — is achieved by a long-horizon control strategy, achieving maximum efficiency of overall grid operations. Most existing approaches [24-28] require a high computational load, are centralized, and need full knowledge of the grid structure and operating conditions. Researchers, in trying to reduce the computational burden, have explored many online economic dispatch strategies for microgrids. These include minimizing the fuel consumption of a microgrid having local heat demands [29], performing an economic dispatch for a microgrid considering renewable energy cost [30], and using a mesh-adaptive direct search method to minimize fuel and 
maintenance costs [31]. However, these methods are still centralized and a priori knowledge of the grid structure is required. Microgrids must be scalable and they are subject to frequent "power source failures" (intermittent renewable power sources). Therefore, decentralized concepts are preferred.

Researchers have proposed several approaches to decentralized power dispatch strategies. De Brabandere [32] presented an optimal power dispatch strategy that requires sharing the marginal cost of each power source. This is carried out through iterative and random communication. Tanaka [33] designed a decentralized control strategy extracted from off-line optimization results. This control design approach, however, requires a priori knowledge of the grid structure and, if the grid structure changes, extensive computations are required. Cagnano [34] suggested an online optimal reactive power control strategy that does not requires a priori knowledge of the grid structure. Implementation, however, requires extensive communications. In this research, we focus on a decentralized power control approach that requires little communication and no a priori knowledge of the grid structure. Past microgrid control work has mainly focused on improving the power quality under fluctuations in generation and load, faults of micro power sources, and low-voltage ride-through. This research, targeting grid efficiency and robustness, addresses a different but equally important problem.

The rest of this paper is organized as follows: the control concept is described in Section 2, and the necessary conditions for the minimum cost are derived in Section 3. A decentralized control algorithm is developed using the necessary conditions in Section 4. The control algorithm is evaluated through simulations in Section 5. Finally, Section 6 presents our conclusions.

\section{Control Concept}

In the islanded microgrid illustrated in Figure 1a, when the terminal voltage values of the power sources are fixed, the control variables are the power outputs of the power sources. By allocating the power outputs while keeping the total demand and total supply balanced, the controller can be tuned to minimize a cost function that include distribution power loss, generation cost, or both. The cost function depends on the active power outputs of the power sources as well as the grid configuration and distribution line impedance values. A meaningful optimal problem is to allocate the power outputs that satisfy the condition for the minimum cost. The necessary condition for the minimum cost, as what has been found in similar problems, is that the marginal costs of all control variables should be the same [35].

Figure 1. (a) Conceptual structure of an islanded microgrid with multiple sources and loads; (b) The equivalent 3-bus model of the microgrid. The symbols in black are measured and the symbols in red are unknowns. $V_{b}$ is a lumped quantity that represents the equivalent voltage of the rest of buses.
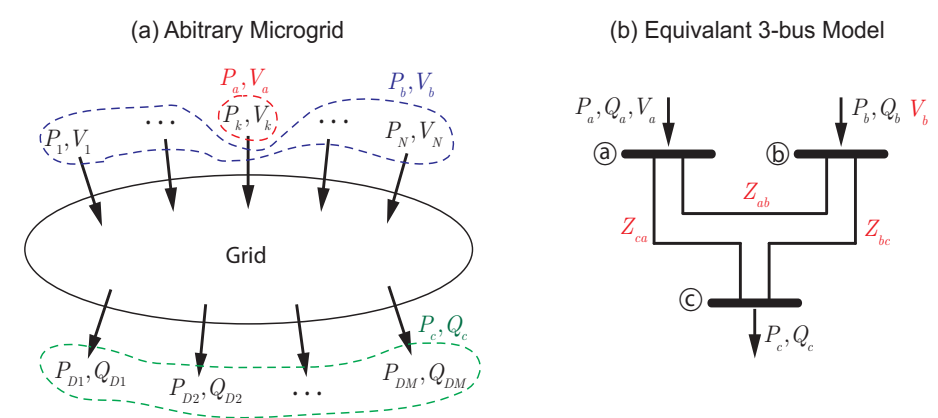
To support plug-and-play functionality and a decentralized operation, the controller should not require a priori knowledge of the grid structure nor direct access to the measurement signals of all power sources. The controller at a power source is thus assumed to have access only to local measurement signals plus a small quantity of information transmitted through communications. Each distributed controller collocated with a power source perceives the overall microgrid system as a 3-bus system consisting of, as shown in Figure 1b, itself, another power source, and a single load — a highly simplified model of the grid. The controller first identifies the parameters of the perceived grid system and then uses the identified model to evaluate the marginal cost. The controllers then make proper adjustments so that the marginal cost evaluated at each controller converges to the same value.

\section{Optimal Control Problem}

\subsection{Problem Formulation}

For a general microgrid shown in Figure 1a, the cost function to be minimized is assumed to be a function of active powers and distribution power losses, as shown in Equation (1). The function $C_{k}\left(P_{k}\right)$ in the first term is the generation cost for active power $P_{k}$ of the $k_{\text {th }}$ power source. The second term is the distribution power loss which is calculated from the total power generation (positive) and the total demand (negative). The parameter $\alpha$ is a weighting factor. The voltage of each power source is assumed to be given and the active power $P_{k}$ are the control variables manipulated to achieve optimality. The equality constraints are the power flow equations and the power balance equations at the demand buses. The cost function is expressed as follows:

$$
\min _{\mathbf{P}}\left[\alpha \sum_{k=1}^{N} C_{k}\left(P_{k}\right)+(1-\alpha)\left(\sum_{k=1}^{N} P_{k}+\sum_{k=N+1}^{N+M} P_{k}\right)\right]
$$

Subject to the following constraints:

$$
\begin{gathered}
P_{k}=f_{k}(\boldsymbol{\delta}, \mathbf{V}), Q_{k}=g_{k}(\boldsymbol{\delta}, \mathbf{V}), k=1,2, \cdots, N+M \\
P_{d}=P_{D}, Q_{d}=Q_{D}
\end{gathered}
$$

where:

$$
\begin{aligned}
& P_{d}=\sum_{k=N+1}^{N+M} P_{k}(\boldsymbol{\delta}, \mathbf{V}) \\
& Q_{d}=\sum_{k=N+1}^{N+M} Q_{k}(\boldsymbol{\delta}, \mathbf{V}) \\
& P_{D}=\sum_{k=N+1}^{N+M} P_{D k} \\
& Q_{D}=\sum_{k=N+1}^{N+M} Q_{D k} \\
& f_{k}: \text { active power equation } \\
& g_{k}: \text { reactive power equation } \\
& \mathbf{P}=\left\{P_{1}, P_{2}, \cdots, P_{N+M}\right\}: \text { active power } \\
& \boldsymbol{\delta}=\left\{\delta_{2}, \delta_{3}, \cdots, \delta_{N+M}\right\}: \text { voltage phase angle }
\end{aligned}
$$


$\mathbf{V}=\left\{V_{1}, V_{2}, \cdots, V_{N+M}\right\}:$ given voltage magnitude

$P_{D k}$ : given active power demand

$Q_{D k}$ : given reactive power demand.

The constraints in Equation (3) are less demanding than balancing the individual power demand, therefore the resulting optimality conditions are still necessary.

\subsection{Necessary Conditions for the Minimum Cost}

We can derive necessary conditions for the minimum cost of Equation (1) using Lagrange multipliers. The Lagrangian function is defined as:

$$
J=\left[\alpha \sum_{k=1}^{N} C_{k}\left(P_{k}\right)+(1-\alpha)\left(\sum_{k=1}^{N} P_{k}+P_{D}\right)\right]+\lambda_{1}\left(P_{d}-P_{D}\right)+\lambda_{2}\left(Q_{d}-Q_{D}\right)
$$

where $\lambda_{1}$ and $\lambda_{2}$ are Lagrange multipliers. The control variables are:

$$
\mathbf{P}=\left\{P_{1}, P_{2}, \cdots, P_{N}\right\}
$$

The necessary conditions for minimal $J$ are as follows:

$$
\begin{gathered}
\frac{\partial J}{\partial P_{k}}=\alpha \frac{\partial C_{k}\left(P_{k}\right)}{\partial P_{k}}+(1-\alpha)+\lambda_{1} \frac{\partial P_{d}}{\partial P_{k}}=0, \text { for } k=1, \cdots, N \\
\frac{\partial J}{\partial \lambda_{1}}=P_{d}-P_{D}=0 \\
\frac{\partial J}{\partial \lambda_{2}}=Q_{d}-P_{D}=0
\end{gathered}
$$

From Equation (6):

$$
\frac{\frac{\partial P_{d}}{\partial P_{k}}}{\alpha \frac{\partial C_{k}\left(P_{k}\right)}{\partial P_{k}}+(1-\alpha)}=-\frac{1}{\lambda_{1}}, \text { for } k=1, \cdots, N
$$

or:

$$
\mu_{1}(t)=\mu_{2}(t)=\cdots=\mu_{N}(t)=\bar{\mu}(t)
$$

where:

$$
\mu_{k}(t) \equiv \frac{\frac{\partial P_{d}}{\partial P_{k}}}{\alpha \frac{\partial C_{k}\left(P_{k}\right)}{\partial P_{k}}+(1-\alpha)}, \text { for } k=1, \cdots, N, \quad \bar{\mu}(t)=\frac{1}{N} \sum_{k=1}^{N} \mu_{k}(t)
$$

The condition shown in Equation (10) has the following physical meaning: the rated marginal cost evaluated at each controller must be equal for the optimal condition to be satisfied. 


\section{Decentralized Controller Design}

\subsection{Controller Design}

The goal of the controller is to satisfy the condition shown in Equation (10). This is achieved by driving all the individual power sources toward the average value of the marginal costs of all nodes. Defining semi-positive definite functions that represent the distance between $\mu_{k}(t)$ and $\bar{\mu}(t)$ as follows:

$$
E_{k}(t)=\frac{1}{2}\left(\mu_{k}(t)-\bar{\mu}(t)\right)^{2}
$$

the condition for the minimum cost, Equation (10), is rewritten:

$$
E_{1}(t)=E_{2}(t)=\cdots=E_{N}(t)=0
$$

Therefore, the controller's goal is:

$$
\lim _{t \rightarrow \infty} E_{k}(t)=0, \forall k
$$

While we know it is not true, we assume that $\partial \bar{\mu}(t) / \partial t \approx 0$, given the fact that the power nodes make their corrections toward the mean, and their actions are largely cancelled by each other. Then the time derivative of Equation (12) becomes:

$$
\frac{\partial E_{k}(t)}{\partial t}=\frac{\partial \mu_{k}(t)}{\partial t}\left(\mu_{k}(t)-\bar{\mu}(t)\right)
$$

If we choose a control law:

$$
\frac{\partial P_{k}}{\partial t}=-\left(\frac{\partial \mu_{k}(t)}{\partial P_{k}}\right)^{-1}\left(\mu_{k}(t)-\bar{\mu}(t)\right)
$$

then:

$$
\frac{\partial \mu_{k}(t)}{\partial t}=\frac{\partial \mu_{k}(t)}{\partial P_{k}} \frac{\partial P_{k}}{\partial t}=-\left(\mu_{k}(t)-\bar{\mu}(t)\right)
$$

From Equation (15), this control laws leads to:

$$
\frac{\partial E_{k}(t)}{\partial t}=-\left(\mu_{k}(t)-\bar{\mu}(t)\right)^{2} \leq 0
$$

If we choose a control law: which means that $E_{k}(t)$ will decrease and $\mu_{k}(t)$ will approach $\bar{\mu}(t)$.

\subsection{Controller Robustness Analysis}

The dynamics of the marginal cost, Equation (17), is exponentially stable when $\partial \bar{\mu}(t) / \partial t=0$. However, when $\bar{\mu}(t)$ is time varying, we need to analyze the stability more rigorously. Considering that $\bar{\mu}(t)$ is the average value of all $\mu_{k}(t), \bar{\mu}(t)$ changes more slowly than $\mu_{k}(t)$, i.e., $\|\dot{\bar{\mu}}(t)\|$ is small. In this case, the stability of the time-varying system can be proven by using Theorem 9.3 in [36]. We can then conclude that $\mu_{k}(t)-\bar{\mu}(t)$ is uniformly, ultimately bounded. Theorem 9.3 in [36] is summarized as follows: 
For a system, $\dot{x}=f(x, u(t)),\|\dot{u}(t)\| \leq \varepsilon$, suppose (i) $f(x, u)$ is locally Lipschitz; (ii) $f(x, u)=0$ has roots $x=h(u)$, and $\|\partial h / \partial u\| \leq L$ holds for all $u$. For the frozen equilibrium point $x=h(a)$, if we define $z=x-h(a)$, where $a$ is a fixed and stable equilibrium point, then we can rewrite the dynamics as $\dot{z}=f[z+h(a), a] \equiv g(z, a)$ Assume that we can find a Lyapunov function $V(z, \alpha)$ that satisfies the conditions:

$$
c_{1}\|z\|^{2} \leq V(z, a) \leq c_{2}\|z\|^{2}, \frac{\partial V}{\partial z} g(z, a) \leq-c_{3}\|z\|^{2},\left\|\frac{\partial V}{\partial z}\right\| \leq c_{4}\|z\|, \quad\left\|\frac{\partial V}{\partial a}\right\| \leq c_{5}\|z\|^{2}
$$

Now, if we consider a change of variable $z=x-h(u)$, then the original system becomes:

$$
\dot{z}=g(z, u)-\frac{\partial h}{\partial u} \dot{u}
$$

For the new system, if:

$$
\varepsilon \leq \frac{c_{1} c_{3}}{c_{2} c_{5}} \times \frac{r}{r+c_{4} L / c_{5}}
$$

then for all $\|z(0)\|<r \sqrt{c_{1} / c_{2}}$, the solutions of Equation (20) are uniformly ultimately bounded by:

$$
\frac{c_{2} c_{4} L \varepsilon}{\left(c_{1} c_{3}-\varepsilon c_{2} c_{5}\right)}
$$

Applying this known Theorem to our case, we can see that if we set $a=\bar{\mu}(t)$ and $V(z, a)=0.5 z^{2}$, then all the parameters can be determined as follows: $c_{1}=c_{2}=0.5, c_{3}=c_{4}=1, c_{5}=0$, and $L=1$. Using these parameters, we can show that the solution of the system dynamics, Equation (17), is bounded by:

$$
\left\|\mu_{k}(t)-\bar{\mu}(t)\right\| \leq \varepsilon
$$

which means that if $\bar{\mu}(t)$ is time varying, then $\mu_{k}(t)$ will approach $\bar{\mu}(t)$ with a steady state error, the value of which depends on the bound of $\|\dot{\bar{\mu}}(t)\|$. If we choose the power control law:

$$
\frac{\partial P_{k}}{\partial t}=-\left(\frac{\partial \mu_{k}(t)}{\partial P_{k}}-\frac{\partial \bar{\mu}(t)}{\partial P_{k}}\right)^{-1}\left(\mu_{k}(t)-\bar{\mu}(t)\right)
$$

the implementation of which requires $\partial \bar{\mu}(t) / \partial P_{k}$, which, if available, results in zero steady state error when $\bar{\mu}(t)$ is time invariant. However, calculating $\partial \bar{\mu}(t) / \partial P_{k}$ at each distributed controller requires complete information of cost as well as the grid structure, which counters the idea of plug-and-play and decentralized implementation. Therefore, we cannot use Equation (24). Instead, we implement the control law Equation (16), which can be implemented locally.

\subsection{Equivalent Grid Model Identification}

To implement the control law, Equation (16), we need to compute $\mu_{k}(t)$ and know $\bar{\mu}(t)$, the later requires transmitting $\mu_{k}(t)$ to all other nodes through a communication network. Calculating $\mu_{k}(t)$ requires knowledge of grid structure due to the partial derivative term, $\partial P_{d} / \partial P_{k}$. While this term is not directly available, it can be approximated if we represent the grid by an equivalent 3-bus model shown 
in Figure $1 b$. The equivalent 3-bus model is sufficient to calculate $\partial P_{d} / \partial P_{k}$, because calculating the partial derivative term requires the relationship only between the active power output of the $k_{\text {th }}$ power source and the total active power demand.

Identification of the equivalent 3-bus model of an arbitrary grid requires the local measurement $\left[P_{a}(t), Q_{a}(t)\right.$, and $\left.V_{a}(t)\right]$ and transmitted information $\left[P_{b}(t), Q_{b}(t), P_{c}(t)\right.$, and $\left.Q_{c}(t)\right]$. At any given time, to determine an equivalent 3-bus model, we need to identify 6 parameters (the resistance and the reactance of three lines) and a variable (the equivalent voltage of Bus $b, V_{b}$ ), as shown in Figure $1 b$. Because we have three independent equations, as shown in Equation (27) and seven unknowns, we cannot uniquely determine the parameters and the variable. Therefore, we accumulate measurements for three time steps, which makes nine independent equations with nine unknowns [six line parameters and $V_{b}(t-2 \Delta t), V_{b}(t-\Delta t)$, and $\left.V_{b}(t)\right]$.

In this time-lapsed solution, the nine unknowns can be solved from a nonlinear optimization problem, as follows:

$$
\min _{X, \theta}\left\|Y_{m}-Y(X, \theta)\right\|^{2}
$$

where:

$$
\begin{aligned}
& Y_{m}=\left[y_{m}(t-2 \Delta t), y_{m}(t-\Delta t), y_{m}(t)\right]^{T} \\
& y_{m}(t)=\left[P_{a}(t), Q_{a}(t), Q_{b}(t)\right] \\
& X=\left[V_{b}(t-2 \Delta t), V_{b}(t-\Delta t), V_{b}(t)\right] \\
& \theta=\left[R_{a b}, R_{b c}, R_{c a}, X_{a b}, X_{b c}, X_{c a}\right] \\
& Y(X, \theta)=\left[\begin{array}{c}
y(X(1), \theta, t-2 \Delta t) \\
y(X(2), \theta, t-\Delta t) \\
y(X(3), \theta, t)
\end{array}\right] \\
& y(x, \theta, t)=\left[p_{a}(x, \theta, t), q_{a}(x, \theta, t), q_{b}(x, \theta, t)\right]^{T}
\end{aligned}
$$

$R$ and $X$ are line resistance and reactance, respectively. The three elements of $y(x, \theta, t)$ are determined by the following equations:

$$
\begin{aligned}
& p_{a}\left(V_{b}(t), \theta, t\right)=\operatorname{Re}\left(S_{a}\left(V_{b}(t), \theta, t\right)\right) \\
& q_{a}\left(V_{b}(t), \theta, t\right)=\operatorname{Im}\left(S_{a}\left(V_{b}(t), \theta, t\right)\right) \\
& q_{b}\left(V_{b}(t), \theta, t\right)=\operatorname{Im}\left(S_{b}\left(V_{b}(t), \theta, t\right)\right)
\end{aligned}
$$

under the equality constraints:

$$
\begin{aligned}
& P_{b}(t)=\operatorname{Re}\left(S_{b}\left(V_{b}(t), \theta, t\right)\right) \\
& P_{c}(t)=\operatorname{Re}\left(S_{c}\left(V_{b}(t), \theta, t\right)\right) \\
& Q_{c}(t)=\operatorname{Im}\left(S_{c}\left(V_{b}(t), \theta, t\right)\right)
\end{aligned}
$$

where:

$$
S_{a}\left(V_{b}(t), \theta, t\right)=\vec{V}_{a}(t)\left(\frac{\vec{V}_{a}(t)-\vec{V}_{b}(t)}{Z_{a b}}+\frac{\vec{V}_{a}(t)-\vec{V}_{c}(t)}{Z_{c a}}\right)
$$




$$
\begin{aligned}
& S_{b}\left(V_{b}(t), \theta, t\right)=\vec{V}_{b}(t)\left(\frac{\vec{V}_{b}(t)-\vec{V}_{a}(t)}{Z_{a b}}+\frac{\vec{V}_{b}(t)-\vec{V}_{c}(t)}{Z_{b c}}\right) \\
& S_{c}\left(V_{b}(t), \theta, t\right)=\vec{V}_{c}(t)\left(\frac{\vec{V}_{c}(t)-\vec{V}_{b}(t)}{Z_{b c}}+\frac{\vec{V}_{c}(t)-\vec{V}_{a}(t)}{Z_{c a}}\right) \\
& \vec{V}_{a}(t)=V_{a}(t) e^{j \delta_{a}(t)} ; \vec{V}_{b}(t)=V_{b}(t) e^{j \delta_{b}(t)} \\
& \vec{V}_{c}(t)=V_{c}(t) e^{j \delta_{c}(t)} ; Z_{a b}=R_{a b}+j X_{a b} \\
& Z_{b c}=R_{b c}+j X_{b c}, Z_{c a}=R_{c a}+j X_{c a}
\end{aligned}
$$

The control law, Equation (16), is implemented in a decentralized way at each power source sequentially. It requires four pieces of information. The first and second pieces are the active and reactive powers generated by all the generators. The third piece is the marginal cost, $\mu_{k}$. The last piece is a token. Only the power source that has the token can adjust its power output. The other power sources that do not have the token adjust their power output to mitigate frequency drooping using a standard proportional control strategy. The flow chart of the control algorithm is shown in Figure 2, and the sequential control concept is shown in Figure 3.

Figure 2. Flow chart of the control algorithm. The variables are defined as follows: $t_{\text {count }}$ is the time counter; $\Delta t$ is the time step; and, $T$ is the control time span.

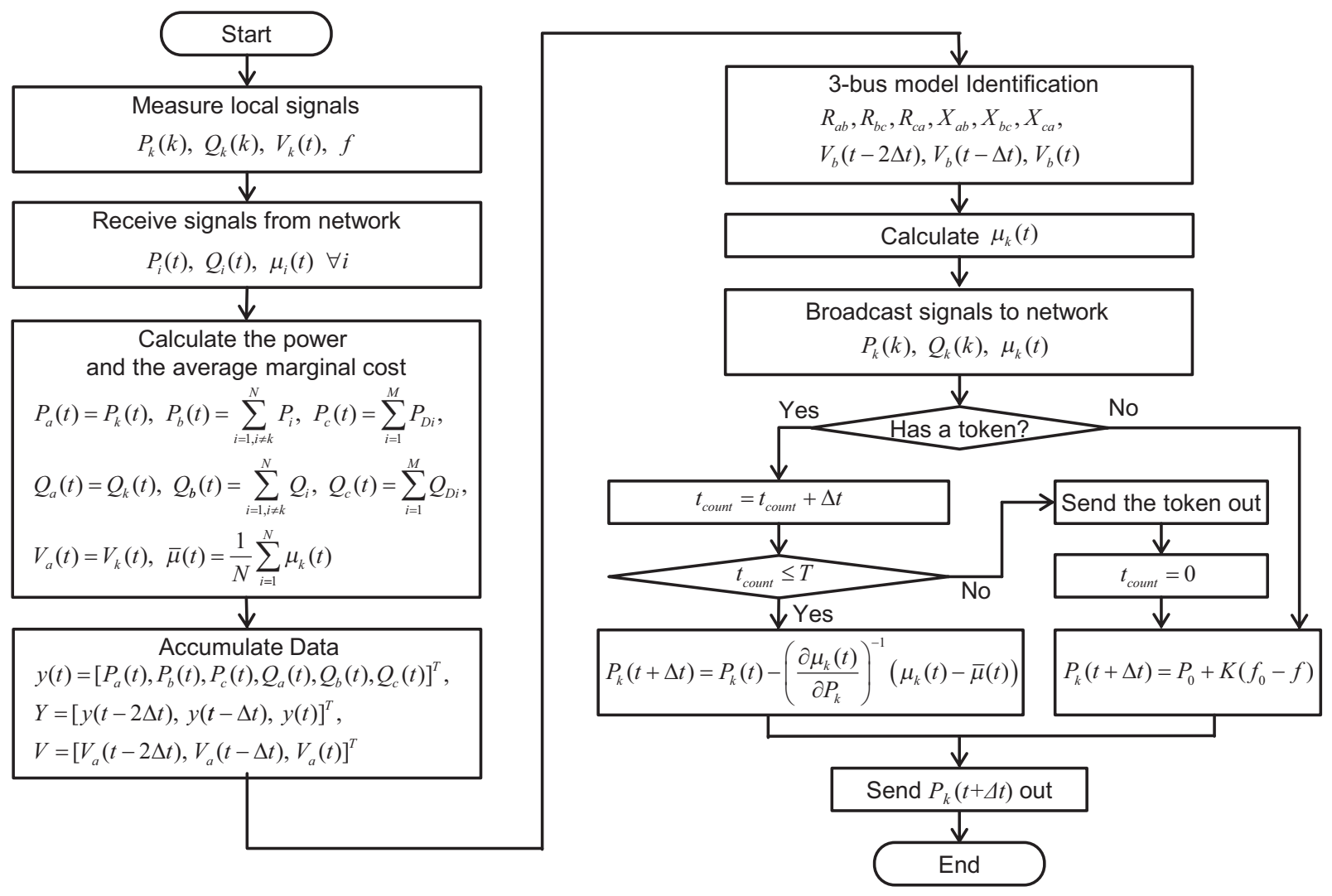


Figure 3. Conceptual control sequences of the decentralized control algorithm. Note that, at each time step, only one power source is controlled by the control law Equation (14), and the other power sources are governed by frequency drooping. $\Delta t$ is a time step and $T$ is a control time span.

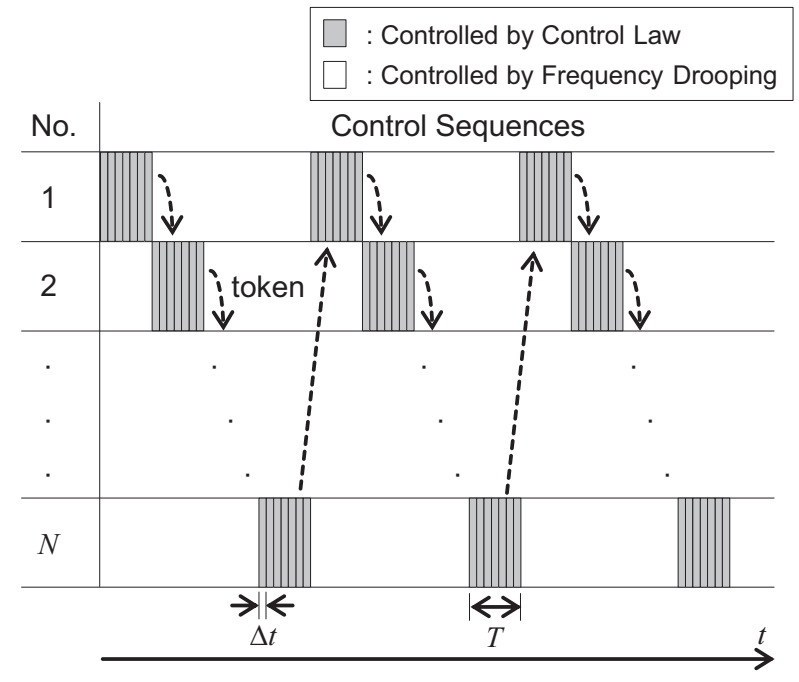

\section{Simulation Results and Discussion}

The performance and flexibility of the control algorithm are assessed through three simulation cases. We created an imagined microgrid with five buses for the first and second cases (Cases 1 and 2); the corresponding grid model is shown in Figure 4. The distribution line parameters and initial conditions are listed in Tables 1 and 2. The cost parameters are listed in Table 3. The last case, Case 3, has six power sources in a 9-bus microgrid shown in Figure 5, and the parameters for the case are described later. For the all cases, the true minimum cost and optimal power outputs were computed by offline computation to provide the reference.

Figure 4. (a) Grid model programmed in Matlab/Simulink ${ }^{\circledR}$ Simpowersystems; (b) Detailed view of the power source block. The power dispatch control algorithm is located in the "High Level Control" block.

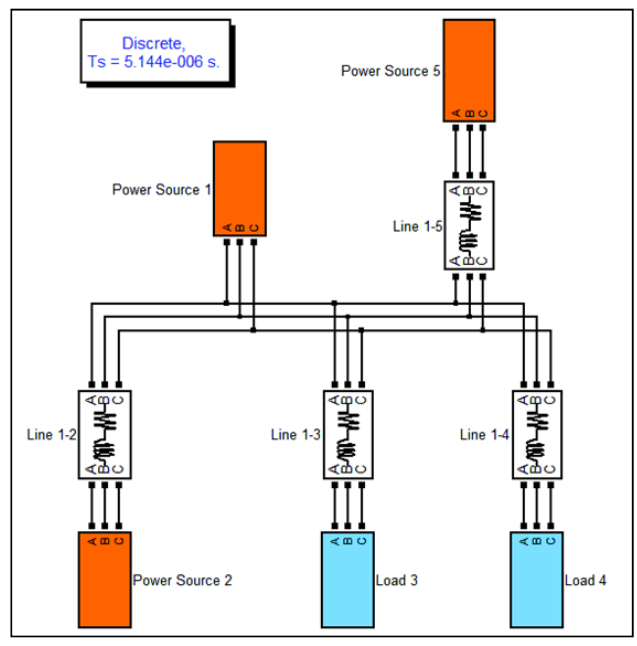

(a)

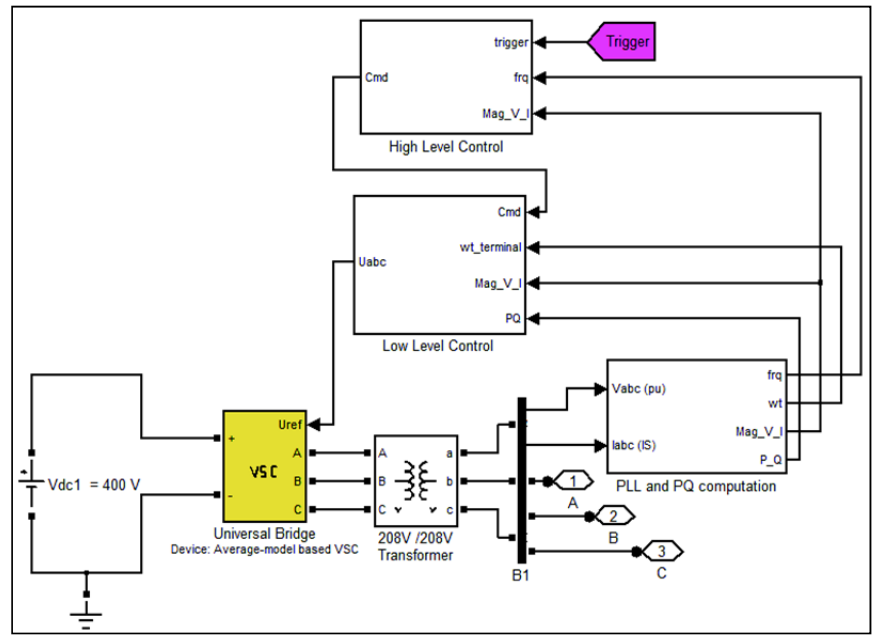

(b) 
Figure 5. Grid model with six power sources and nine buses.

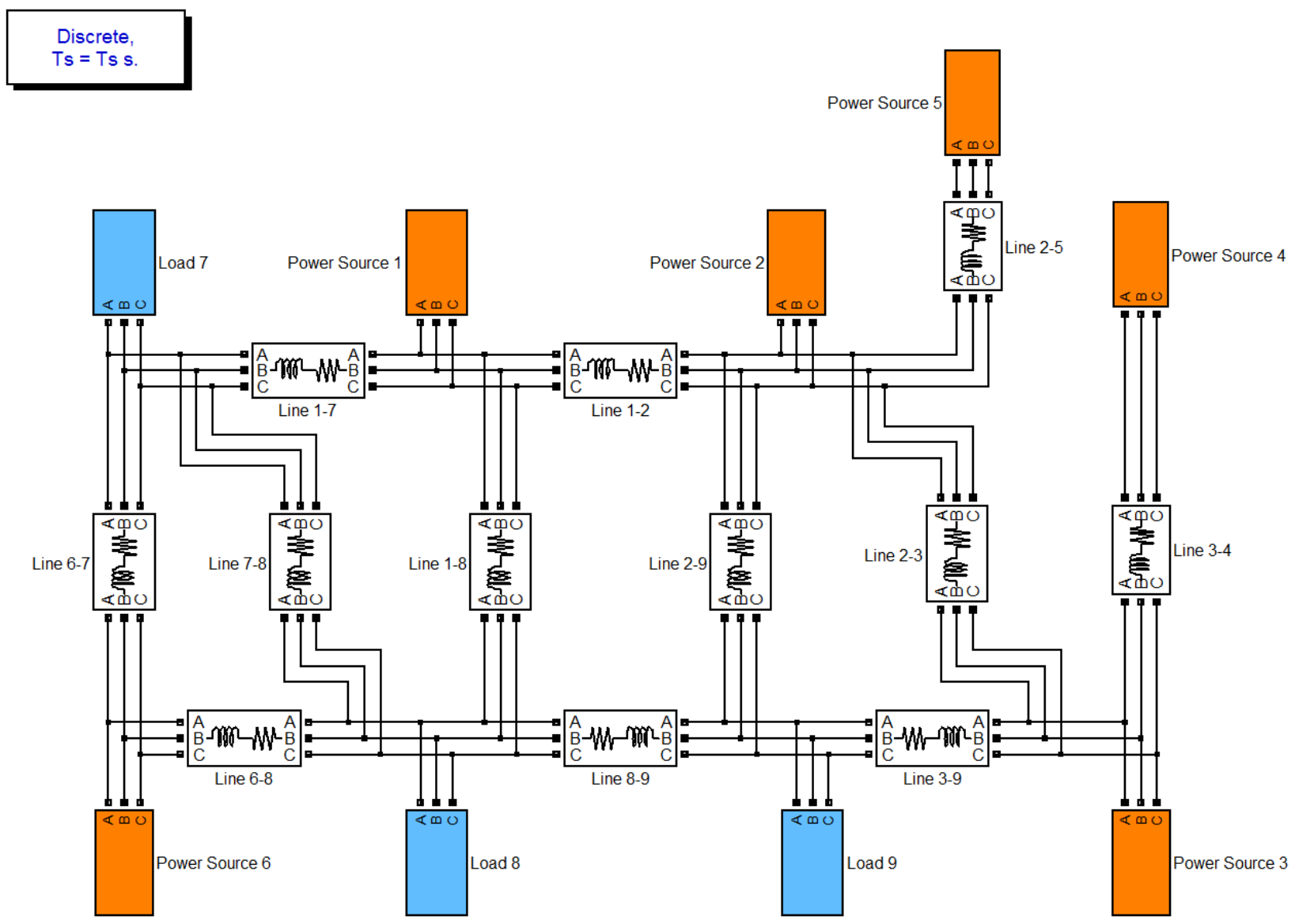

Table 1. Grid parameters.

\begin{tabular}{cccc}
\hline Symbol & Value & Unit & Description \\
\hline$R_{12}, R_{14}, R_{15}$ & 0.1 & $\mathrm{pu}$ & Line resistance \\
$R_{13}$ & 0.15 & $\mathrm{pu}$ & Line resistance \\
$X_{12}, X_{14}, X_{15}$ & 0.01 & $\mathrm{pu}$ & Line reactance \\
$X_{13}$ & 0.015 & $\mathrm{pu}$ & Line reactance \\
\hline
\end{tabular}

The base voltage is $110 \mathrm{~V}$, the base power is $60 \mathrm{~kW}$.

Table 2. Initial conditions for simulation.

\begin{tabular}{cccc}
\hline Symbol & Value & Unit & Description \\
\hline$P_{L 3}, P_{L 4}$ & $0.35,0.3$ & $\mathrm{pu}$ & Active power demand at Buses 3 and 4, constant \\
$Q_{L 3}, Q_{L 4}$ & $0.07,0.06$ & $\mathrm{pu}$ & Reactive power demand at Buses 3 and 4, constant \\
$P_{G 1}$ & 0.3341 & $\mathrm{pu}$ & Active power from power source 1 \\
$P_{G 2}$ & 0.3068 & $\mathrm{pu}$ & Active power from power source 2 \\
$P_{G 5}$ & 0.1206 & $\mathrm{pu}$ & Active power from power source 5 \\
$V_{1}$ & 1.0 & $\mathrm{pu}$ & Voltages of Bus 1, constant \\
$V_{2}$ & 1.02 & $\mathrm{pu}$ & Voltages of Bus 2, constant \\
$V_{5}$ & 1.015 & $\mathrm{pu}$ & Voltages of Bus 5, constant \\
\hline
\end{tabular}

The base voltage is $110 \mathrm{~V}$; the base power is $60 \mathrm{~kW}$. As the controllers are engaged, $P_{G 1}, P_{G 2}$, and $P_{G 5}$ vary. 
Table 3. Cost parameters for simulation.

\begin{tabular}{ccccc}
\hline Cases & $\boldsymbol{\alpha}$ & $\boldsymbol{C}_{\mathbf{1}}\left(\boldsymbol{P}_{\mathbf{1}}\right)$ & $\boldsymbol{C}_{\mathbf{2}}\left(\boldsymbol{P}_{\mathbf{2}}\right)$ & $\boldsymbol{C}_{\mathbf{5}}\left(\boldsymbol{P}_{\mathbf{5}}\right)$ \\
\hline Case 1 & 0.5 & $0.05 \times P_{1}$ & $0.0525 \times P_{2}$ & $0.055 \times P_{3}$ \\
Case 2 & 0.0 & - & - & - \\
\hline
\end{tabular}

In Case 1, the weighting factor $\alpha$ is set to 0.5 , to minimize the combination of the generation cost and distribution losses. This represents the situations when the power sources consume different types of fuels or have different efficiencies. Figure $6 \mathrm{a}$ shows the performance of the decentralized power control algorithm. The first plot shows power outputs from all power sources in solid lines and their optimal values in dashed lines. The second plot shows the rated marginal cost $\mu_{k}$ defined in Equation (11). The last plot shows the cost compared to the minimum cost calculated by off-line optimization. The optimal power flow solution ( $P 1_{\text {opt }}, P 2_{\text {opt }} P 5_{\text {opt }}$ in the top plot) shows that the power from Power Source 1 is the highsest and from Power Source 5 is the lowest. This is because the line resistance from Power Source 1 to the load buses is the lowsest $(0.1 \mathrm{pu})$ and the generating cost of Power Souce 1 is the lowest. To minimize the generation cost and the distribution loss, the controllers allocate the highest power output to Power Souce 1. Each controller moves its marginal cost toward the averaged marginal cost. We can see that the three marginal values finally convege to a common value and the power outputs also move toward the optimal values.

In Case 2, the weighting factor $\alpha$ is set to zero and thus all the generating costs are ignored, resulting in a distribution loss minimization problem. This case represents the case with zero or very low generating cost, such as solar panels. Figure $6 \mathrm{~b}$ shows the performance of the decentralized power control algorithm. Because the line resistence, from Power Source 1 to the load buses is the lowest, Power Source 1 provides highest power to the load buses.

Figure 6. (a) Performance of the decentralized control algorithm of Case 1; (b) Performance of the decentralized control algorithm of Case 2.
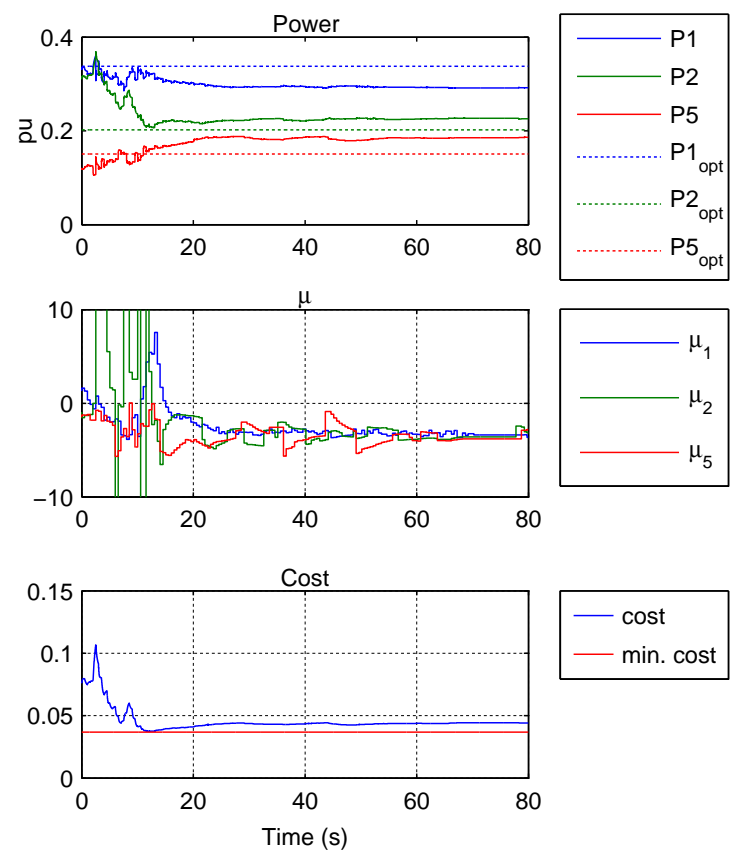

(a)
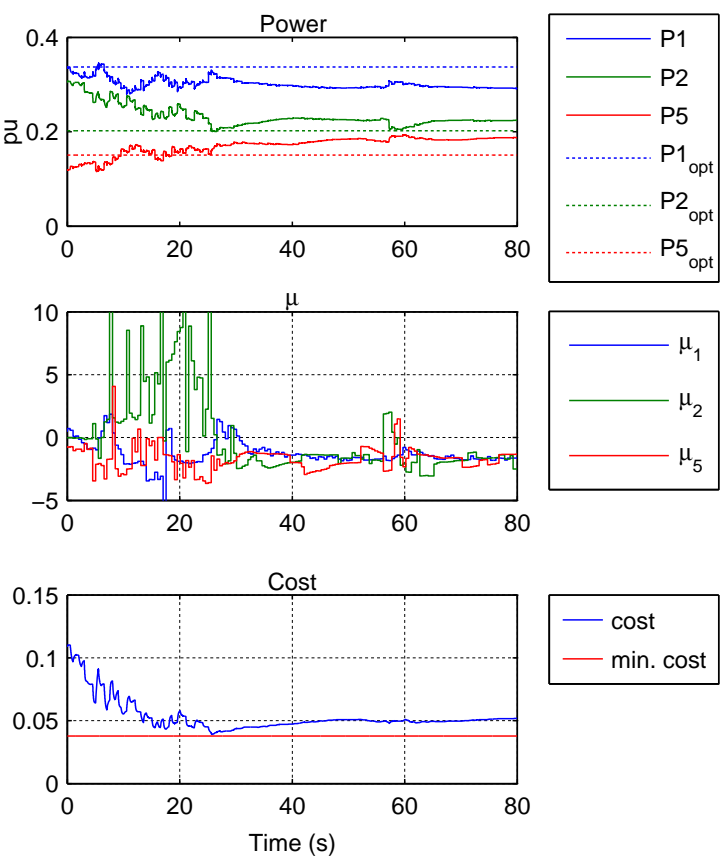

(b) 
In the two cases above, each controller moves its marginal cost toward the averaged marginal cost. The marginal costs fluctuate significantly and finally converge to a negative value. The positive marginal cost $\left(\mu_{k}>0\right)$ means that the total cost increases as the local power increases, which happens when its power ouputs are too high and thus the power loss is excessive. Therefore, every controller tries to minimize the rated marginal cost and implicit negotiation occurs through broadcating their marginal cost. The equilibrium or minimal cost is achieved when every controll has equal marginal cost. The cost is reduced significantly with time. The cost, however, does not converge to the minimum cost completely. This is because the maginal costs are not exactly the same. This is the performance limitation analyzed in Section IV, the analysis of which shows that $\mu_{k}(t)-\bar{\mu}(t)$ may have an error due to error in model identification and $\bar{\mu}(t)$ is time-varying.

The scalability and flexibility of the control algorithm are tested using Case 3, which has a very different grid configuration. Figure 7 shows a microgrid with nine buses. In this case, $\alpha$ is set as zero and thus all the generating costs are ignored, resulting in a distribution loss minimization problem. As shown in Figure 7, the control concept based on the three-bus approximation still works well even when the actual grid is a lot more complicated, which seems to show that it has some robustness in working in expanded grid structure.

Figure 7. Performance of the decentralized control algorithm of Case 3.
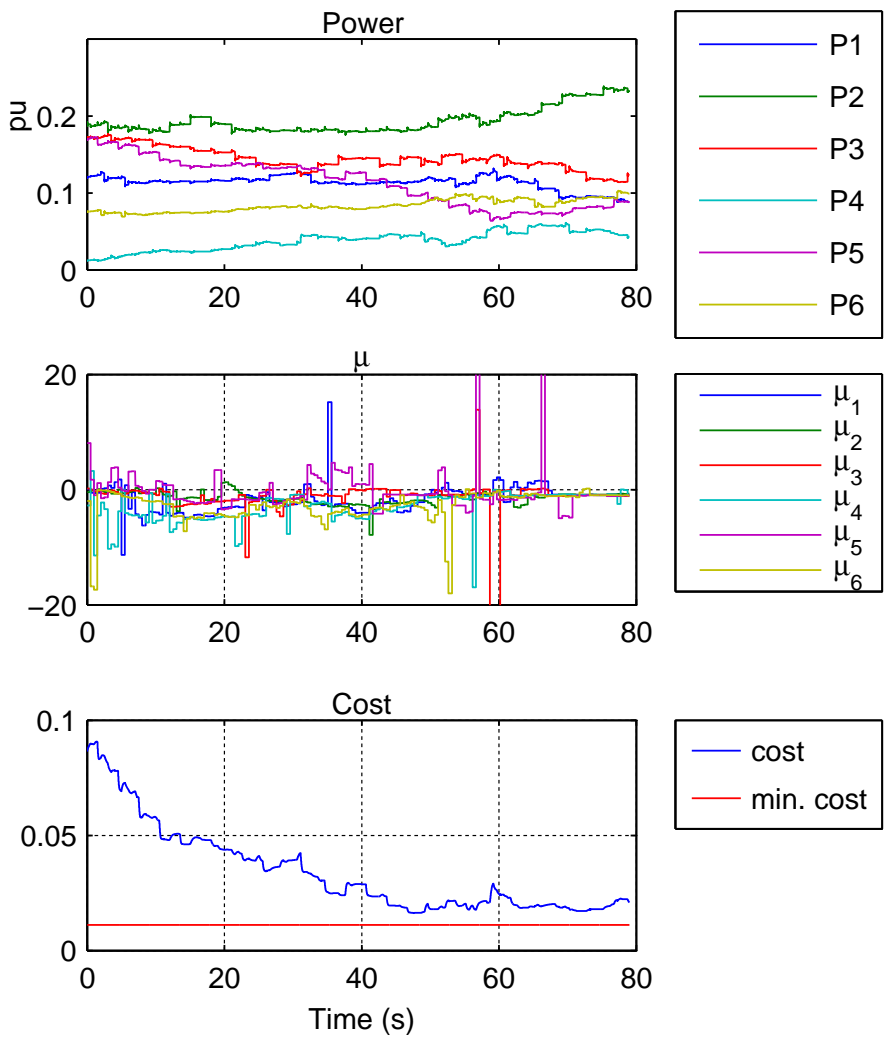

\section{Conclusions}

In this paper we have proposed a decentralized power dispatch algorithm that minimizes a user-defined cost that can include weighted power generation cost and distribution loss. The algorithm is designed to work in a decentralized fashion, requiring only a small amount of information to be 
communicated: power generated at each node, and a token to ensure only one power node is active at any given moment. The control law is derived from the necessary conditions for the minimum cost, and each node works to reduce its deviation from the average marginal cost it perceives. The algorithm does not require a priori knowledge of the grid structure. This is achieved by using a 3-bus model to represent the overall grid, and through system identification, each power node has a perceived grid behavior. Computer-based simulation showed that the algorithm works well both for a 5-node microgrid and a 9-node grid.

\section{Acknowledgments}

This work was supported by the Automotive Research Center (ARC), a U.S. Army center of excellence in modeling and simulation of ground vehicles.

\section{Conflicts of Interest}

The authors declare no conflict of interest.

\section{References}

1. Peças Lopes, J.A.; Polenz, S.A.; Moreira, C.L.; Cherkaoui, R. Identification of control and management strategies for LV unbalanced microgrids with plugged-in electric vehicles. Electr. Power Syst. Res. 2010, 80, 898-906.

2. Peças Lopes, J.A.; Moreira, C.L.; Madureira, A.G. Defining control strategies for microgrids islanded operation. IEEE Trans. Power Syst. 2006, 21, 916-924.

3. Lidula, N.W.A.; Rajapakse, A.D. Microgrids research: A review of experimental microgrids and test systems. Renew. Sustain. Energy Rev. 2011, 15, 186-202.

4. Guerrero, J.M.; de Vicuna, L.G.; Matas, J.; Castilla, M.; Miret, J. A wireless controller to enhance dynamic performance of parallel inverters in distributed generation systems. IEEE Trans. Power Electron. 2004, 19, 1205-1213.

5. Green, T.C.; Prodanović, M. Control of inverter-based micro-grids. Electr. Power Syst. Res. 2007, $77,1204-1213$.

6. Jiayi, H.; Chuanwen, J.; Rong, X. A review on distributed energy resources and MicroGrid. Renew. Sustain. Energy Rev. 2008, 12, 2472-2483.

7. Lasseter, R.; Akhil, A.; Marnay, C.; Stephens, J.; Dagle, J.; Guttromson, R.; Meliopoulous, A.S.; Yinger, R.; Eto, J. Integration of Distributed Energy Resources: The CERTS Microgrid Concept; LBNL-50829; U.S. Department of Energy: Washington, DC, USA; and California Energy Commission: Sacramento, CA, USA, 2002.

8. Hatziargyriou, N.; Asano, H.; Iravani, R.; Marnay, C. Microgrids. IEEE Power Energy Mag. 2007, 5, 78-94.

9. Lasseter, R.H.; Paigi, P. Microgrid: A Conceptual Solution. In Proceedings of the 2004 IEEE 35th Annual Power Electronics Specialists Conference, Aachen, Germany, 20-25 June 2004; pp. $4285-4290$. 
10. Guerrero, J.M. Microgrids: Integration of Distributed Energy Resources into the Smart-Grid. In Proceedings of the 2010 IEEE International Symposium on Industrial Electronics(ISIE), Bari, Italy, 4-7 July 2010; pp. 4281-4414.

11. Kempton, W.; Tomic, J. Vehicle-to-grid power implementation: From stabilizing the grid to supporting large-scale renewable energy. J. Power Sources 2005, 144, 280-294.

12. Ersal, T.; Ahn, C.; Hiskens, I.A.; Peng, H.; Stein, J.L. Impact of Controlled Plug-in EVs on Microgrids: A Military Microgrid Example. In Proceedings of the 2011 IEEE Power and Energy Society General Meeting, Detroit, MI, USA, 24-29 July 2011; pp. 1-7.

13. Ahn, C.; Li, C.-T.; Peng, H. Optimal decentralized charging control algorithm for electrified vehicles connected to smart grid. J. Power Sources 2011, 196, 10369-10379.

14. Kempton, W.; Tomic, J. Vehicle-to-grid power fundamentals: Calculating capacity and net revenue. J. Power Sources 2005, 144, 268-279.

15. Tomic, J.; Kempton, W. Using fleets of electric-drive vehicles for grid support. J. Power Sources 2007, 168, 459-468.

16. Ahn, C.; Peng, H. Decentralized voltage control to minimize distribution power loss of microgrids. IEEE Trans. Smart Grid 2013, 4, 1297-1304.

17. Vojdani, A. Smart integration. IEEE Power Energy Mag. 2008, 6, 71-79.

18. Amin, M.A.; Wollenberg, B.F. Toward a smart grid: Power delivery for the 21 st century. IEEE Power Energy Mag. 2005, 3, 34-41.

19. De Brabandere, K.; Bolsens, B.; van den Keybus, J.; Woyte, A.; Driesen, J.; Belmans, R. A Voltage and Frequency Droop Control Method for Parallel Inverters. In Proceedings of the 2004 IEEE 35th Annual Power Electronics Specialists Conference, Aachen, Germany, 20-25 June 2004; pp. 2501-2507.

20. Tokudome, M.; Tanaka, K.; Senjyu, T.; Yona, A.; Funabashi, T.; Chul-Hwan, K. Frequency and Voltage Control of Small Power Systems by Decentralized Controllable Loads. In Proceedings of the International Conference on Power Electronics and Drive Systems (PEDS 2009), Taipei, Taiwan, 2-5 November 2009; pp. 666-671.

21. Guerrero, J.M.; Vasquez, J.C.; Matas, J.; de Vicuna, L.G.; Castilla, M. Hierarchical control of droop-controlled AC and DC microgrids-A general approach toward standardization. IEEE Trans. Ind. Electron. 2011, 58, 158-172.

22. Engler, A.; Soultanis, N. Droop Control in LV-Grids. In Proceedings of the International Conference on Future Power Systems, Amsterdam, The Netherlands, 18 November 2005; pp. 1-6.

23. Katiraei, F.; Iravani, M.R. Power management strategies for a microgrid with multiple distributed generation units. IEEE Trans. Power Syst. 2006, 21, 1821-1831.

24. Kishore, A.; Hill, E.F. Static optimization of reactive power sources by use of sensitivity parameters. IEEE Trans. Power Appar. Syst. 1971, PAS-90, 1166-1173.

25. Sun, D.T.W.; Shoults, R.R. A preventive strategy method for voltage and reactive power dispatch. IEEE Trans. Power Appar. Syst. 1985, PAS-104, 1670-1676.

26. Granville, S. Optimal reactive dispatch through interior point methods. IEEE Trans. Power Syst. 1994, 9, 136-146. 
27. Yoshida, H.; Kawata, K.; Fukuyama, Y.; Takayama, S.; Nakanishi, Y. A particle swarm optimization for reactive power and voltage control considering voltage security assessment. IEEE Trans. Power Syst. 2000, 15, 1232-1239.

28. Yoo, C.-H.; Chung, I.-Y.; Lee, H.-J.; Hong, S.-S. Intelligent control of battery energy storage for multi-agent based microgrid energy management. Energies 2013, 6, 4956-4979.

29. Hernandez-Aramburo, C.A.; Green, T.C.; Mugniot, N. Fuel consumption minimization of a microgrid. IEEE Trans. Ind. Appl. 2005, 41, 673-681.

30. Augustine, N.; Suresh, S.; Moghe, P.; Sheikh, K. Economic Dispatch for a Microgrid Considering Renewable Energy Cost Functions. In Proceedings of the IEEE PES Innovative Smart Grid Technologies, Washington, DC, USA, 16-20 January 2012; pp. 1-7.

31. Mohamed, F.A.; Koivo, H.N. System modelling and online optimal management of microgrid using mesh adaptive direct search. Int. J. Electr. Power Energy Syst. 2010, 32, 398-407.

32. De Brabandere, K.; Vanthournout, K.; Driesen, J.; Deconinck, G.; Belmans, R. Control of Microgrids. In Proceedings of the IEEE Power Engineering Society General Meeting, Tampa, FL, USA, 24-28 June 2007; pp. 1-7.

33. Tanaka, K.; Senjyu, T.; Toma, S.; Yona, A.; Funabashi, T.; Kim, C.-H. Decentralized Voltage Control in Distribution Systems by Controlling Reactive Power of Inverters. In Proceedings of the IEEE International Symposium on Industrial Electronics, Seoul, Korea, 5-8 July 2009; pp. 1385-1390.

34. Cagnano, A.; de Tuglie, T.; Liserre, M.; Mastromauro, R.A. Online optimal reactive power control strategy of PV-inverters. IEEE Trans. Ind. Electron. 2011, 58, 4549-4558.

35. Papalambros, P.Y.; Wilde, D.J. Principles of Optimal Design: Modeling and Computation, 2nd ed.; Cambridge University Press: Cambridge, UK, 2000; pp. 194-197

36. Khalil, H.K. Stability of Perturbed Systems. In Nonlinear Systems, 3rd ed.; Prentice Hall: Upper Saddle River, NJ, USA, 2002.

(C) 2013 by the authors; licensee MDPI, Basel, Switzerland. This article is an open access article distributed under the terms and conditions of the Creative Commons Attribution license (http://creativecommons.org/licenses/by/3.0/). 